\title{
Stability Estimates for an Inverse Problem for the Linear Boltzmann Equation
}

\author{
Rolci Cipolatti, Carlos M. Motta, \\ and Nilson C. ROBERTY
}

\author{
Departamento de Métodos Matemáticos \\ Instituto de Matemática \\ Universidade Federal do Rio de Janeiro \\ C.P. 68530 , CEP 21945-970 \\ Rio de Janeiro - Brazil \\ cipolatti@im.ufrj.br
}

ICE/P1/DEMAT

Universidade Federal Rural do Rio de Janeiro

Rio de Janeiro - Brazil

drummath@yahoo.com

Programa de Engenharia Nuclear

COPPE, Universidade Federal do Rio de Janeiro

C.P. 68509 , CEP 21945-970

Rio de Janeiro - Brazil

nilson@con.ufrj.br

Received: March 18, 2005

Accepted: June 14, 2005

\begin{abstract}
In this paper we consider the inverse problem of recovering the total extinction coefficient and the collision kernel for the time-dependent Boltzmann equation via boundary measurements. We obtain stability estimates for the extinction coefficient in terms of the albedo operator and also an identification result for the collision kernel.

Key words: inverse problems, albedo operator, stability estimates.

2000 Mathematics Subject Classification: 35R30, 83D75.
\end{abstract}

\section{Introduction}

In this paper we consider an inverse problem for the linear Boltzmann equation

$$
\partial_{t} u+\omega \cdot \nabla_{x} u+q u=K_{f}[u] \quad \text { in }(0, T) \times S \times \Omega,
$$


where $T>0, \Omega$ is a bounded and convex domain of $\mathbb{R}^{N}, N \geq 2, S=\mathbb{S}^{N-1}$ denotes the unit sphere of $\mathbb{R}^{N}, q \in L^{\infty}(\Omega)$ and $K_{f}$ is the integral operator with kernel $f\left(x, \omega^{\prime}, \omega\right)$ defined by

$$
K_{f}[u](t, \omega, x)=\int_{S} f\left(x, \omega^{\prime}, \omega\right) u\left(t, \omega^{\prime}, x\right) d \omega^{\prime} .
$$

In applications $(N=2$ or 3$)$, (1) describes the dynamics of a monokinetic flow of particles in a body $\Omega$ under the assumption that the interaction between them is negligible (which leads us to discard nonlinear terms). For instance, in the case of a low-density flux of neutrons (see $[7,13]), q \geq 0$ is the total extinction coefficient and the collision kernel $f$ is given by

$$
f\left(x, \omega^{\prime}, \omega\right)=q(x) c(x) h\left(x, \omega^{\prime} \cdot \omega\right),
$$

where the coefficient $c$ corresponds to the within-group scattering probability and the kernel $h$ describes the anisotropy of the scattering process. In this case, $q(x) u(t, \omega, x)$ describes the loss of particles at $x$ in the direction $\omega$ at time $t$ due to absorption or scattering, while the integral on the right hand side of (1) represents the production of particles at $x$ in the direction $\omega$ from those coming from directions $\omega^{\prime}$.

The inverse problem associated with (1) that we are interested here is recovering $q$ and $f$ by boundary measurements. That will lead us to consider the albedo operator $\mathcal{A}$, that maps the incoming flux on the boundary $\partial \Omega$ into the outgoing one.

There is a lot of papers devoted to this problem and we specially mention the general results for the identification one, obtained by Choulli and Stefanov [5] (see also [11]), that state that $q$ and $f$ are uniquely determined by $\mathcal{A}$. There is also a wide bibliography concerning the stationary case (See for instance those by V. G. Romanov [14, 15], P. Stefanov and G. Uhlmann [16], Tamasan [17], J. N. Wang [18], and also the references therein.)

In this paper, we are mainly concerned with stability estimates for the timedependent equation (1). We consider $q_{j}, f_{j}, j=1,2$, and

$$
\mathcal{A}_{j}: L^{1}\left(0, T ; L^{1}\left(\Sigma^{-} ; d \xi\right)\right) \rightarrow L^{1}\left(0, T ; L^{1}\left(\Sigma^{+} ; d \xi\right)\right)
$$

the corresponding albedo operator (that will be precisely described in section 2 below, as well as the spaces concerned). Since $\mathcal{A}_{j}$ is linear and bounded, we consider the usual norm $\left\|\mathcal{A}_{j}\right\|_{1}$.

Our main result is the following:

Theorem 1.1. We assume that $\left\|q_{j}\right\|_{\infty} \leq M$ for some $M>0$ and $f_{j} \in L^{\infty}(\Omega$; $\left.L^{2}(S \times S)\right), j=1,2$. If $T>\operatorname{diam}(\Omega)$, there exists $C=C(M)>0$ such that

$$
\left\|q_{1}-q_{2}\right\|_{H^{-\frac{1}{2}}(\Omega)} \leq C\left\|\mathcal{A}_{1}-\mathcal{A}_{2}\right\|_{1}
$$

Moreover, if $q_{1}, q_{2} \in H^{\frac{N}{2}+s}(\Omega)$ for some $s>0$ and $\left\|q_{j}\right\|_{H^{\frac{N}{2}+s}(\Omega)} \leq M, j=1,2$, then, for each $0<r<s$, there exists $C_{r}>0$ such that

$$
\left\|q_{1}-q_{2}\right\|_{H^{\frac{N}{2}+r}(\Omega)} \leq C_{r}\left\|\mathcal{A}_{1}-\mathcal{A}_{2}\right\|_{1}^{\theta(r)}
$$


where $\theta(r)=2(s-r) /(N+2 s+1)$. In particular, for each $0<r<s$ there exists $\tilde{C}_{r}$ such that

$$
\left\|q_{1}-q_{2}\right\|_{\infty} \leq \tilde{C}_{r}\left\|\mathcal{A}_{1}-\mathcal{A}_{2}\right\|_{1}^{\theta(r)}
$$

Using the same ideas considered in the proof of Theorem 1.1, we obtain the following partial result on the identification of both $q$ and $f$ in (1):

Theorem 1.2. Let $q_{1}, q_{2} \in L^{\infty}(\Omega)$ and $f_{1}, f_{2} \in L^{\infty}\left(\Omega ; L^{2}(S \times S)\right)$. If $T>\operatorname{diam}(\Omega)$ and $\mathcal{A}_{1}=\mathcal{A}_{2}$, then $q_{1}=q_{2}$. Moreover, if $f_{j}\left(x, \omega^{\prime}, \omega\right)=g_{j}(x) h\left(\omega^{\prime}, \omega\right)$, with $h \in$ $L^{\infty}(S \times S)$ such that $h(\omega, \omega) \neq 0$, a.e. on $S$, then $g_{1}=g_{2}$.

We organize the paper as follows: in section 2 we introduce the functional framework in which the initial-boundary value problem for (1) is well posed in the sense of the semigroup theory and where the albedo operator is defined over suitable $L^{p}$ spaces; in section 3, following an argument used in [18] and an analogous strategy used for identifying coefficients in the wave equation (see $[6,12]$ ), we construct special oscillatory solutions of (1) that allow us to prove Theorem 1.1. Although the result established in the Theorem 1.2 is not new, we also present here its proof to highlight the method.

\section{Functional framework and well-posedness results}

For the reader's convenience we gather below a few more or less well known results concerning the functional framework for the operator $\omega \cdot \nabla_{x}$ and the semigroup it generates.

Let $\Omega \subset \mathbb{R}^{N}(N \geq 2)$ be a convex and bounded domain of class $C^{1}$ and $S=\mathbb{S}^{N-1}$ the unit sphere of $\mathbb{R}^{\bar{N}}$. We denote by $Q=S \times \Omega$ and $\Sigma$ its boundary, i.e., $\Sigma=S \times \partial \Omega$. For $p \in[1,+\infty)$ we consider the space $L^{p}(Q)$ with the usual norm

$$
\|u\|_{L^{p}(Q)}=\left(\int_{Q}|u(\omega, x)|^{p} d x d \omega\right)^{1 / p}
$$

where $d \omega$ denotes the surface measure on $S$ associated to the Lebesgue measure in $\mathbb{R}^{N-1}$.

For each $u \in L^{p}(Q)$ we define $A_{0} u$ by

$$
\left(A_{0} u\right)(\omega, x)=\omega \cdot \nabla_{x} u(\omega, x)=\sum_{k=1}^{N} \omega_{k} \frac{\partial u}{\partial x_{k}}(\omega, x), \quad \omega=\left(\omega_{1}, \ldots, \omega_{N}\right)
$$

where the derivatives are taken in the sense of distributions in $\Omega$.

One checks easily that setting $\mathcal{W}_{p}=\left\{u \in L^{p}(Q) ; A_{0} u \in L^{p}(Q)\right\}$, the operator $\left(A_{0}, \mathcal{W}_{p}\right)$ is a closed densely defined operator and $\mathcal{W}_{p}$ with the graph norm is a Banach space. 
For every $\sigma \in \partial \Omega$, we denote $\nu(\sigma)$ the unit outward normal at $\sigma \in \partial \Omega$ and we consider the sets

$$
\Sigma^{ \pm}=\{(\omega, \sigma) \in S \times \partial \Omega ; \pm \omega \cdot \nu(\sigma)>0\} .
$$

We also denote $\Sigma_{\omega}^{ \pm}=\left\{\sigma \in \partial \Omega ;(\omega, \sigma) \in \Sigma^{ \pm}\right\}$(respectively, the incoming and outcoming boundaries in the direction $\omega)$.

Remark. It is well known that functions $u \in \mathcal{W}_{p}$ may present singularities on $\Sigma$ at points $(\omega, \sigma)$ such that $\omega \cdot \nu(\sigma)=0$, in such a way that $u_{\left.\right|_{\Sigma}} \notin L^{p}(\Sigma)$. For instance, consider $\alpha>0, \Omega=\left\{\left(x_{1}, x_{2}\right) \in \mathbb{R}^{2} ; x_{1}^{2}+x_{2}^{2}<1\right\}$ and $u: S \times \Omega \rightarrow \mathbb{R}$ defined by

$$
u(\omega, x)=\frac{1}{\left(\omega_{2} x_{1}-\omega_{1} x_{2}+1\right)^{\alpha}} .
$$

It is easy to check that if $\alpha<3 / 4$ one has $u \in \mathcal{W}_{2}$ but $u_{\Sigma_{\Sigma^{+}}} \notin L^{2}\left(\Sigma^{+}\right)$. Yet worse, if $\alpha \in[1 / 2,3 / 4)$ one has

$$
\int_{\Sigma^{+}}(\omega \cdot \nu(\sigma))|u(\omega, \sigma)|^{2} d \omega d \sigma=+\infty
$$

In order to well define the albedo operator as a trace operator on the outcoming boundary, we consider $L^{p}\left(\Sigma^{ \pm} ; d \xi\right)$, where $d \xi=|\omega \cdot \nu(\sigma)| d \sigma d \omega$, and we introduce the spaces

$$
\widetilde{\mathcal{W}}_{p}^{ \pm}=\left\{u \in \mathcal{W}_{p} ; u_{\left.\right|_{\Sigma^{ \pm}}} \in L^{p}\left(\Sigma^{ \pm} ; d \xi\right)\right\},
$$

which are Banach spaces if equipped with the norms

$$
\|u\|_{\widetilde{\mathcal{W}}_{p}^{ \pm}}=\left(\|u\|_{\mathcal{W}_{p}}^{p}+\int_{\Sigma^{ \pm}}|\omega \cdot \nu(\sigma) \| u(\omega, \sigma)|^{p} d \sigma d \omega\right)^{1 / p}
$$

The next two lemmas concern the continuity and surjectivity of the trace operators $($ see $[3,4])$ :

$$
\gamma_{ \pm}: \widetilde{\mathcal{W}}_{p}^{ \pm} \rightarrow L^{p}\left(\Sigma^{\mp} ; d \xi\right), \quad \gamma_{ \pm}(u)=u_{\left.\right|_{\Sigma \mp}}
$$

Lemma 2.1. Let $1 \leq p<+\infty$. Then there exists $C>0$ (depending only on $p$ ) such that

$$
\int_{\Sigma^{\mp}}\left|\omega \cdot \nu(\sigma)\left\|\left.u(\omega, \sigma)\right|^{p} d \sigma d \omega \leq C\right\| u \|_{\widetilde{\mathcal{W}}_{p}^{ \pm}}^{p}, \quad \forall u \in \widetilde{\mathcal{W}}_{p}^{ \pm} .\right.
$$

Moreover, if $p>1$ and $1 / p+1 / p^{\prime}=1$, we have the Gauss identity

$$
\int_{Q} \operatorname{div}_{x}(u v \omega) d x d \omega=\int_{\Sigma} \omega \cdot \nu(\sigma) u(\omega, \sigma) v(\omega, \sigma) d \sigma d \omega,
$$

for all $u \in \widetilde{\mathcal{W}}_{p}^{ \pm}$and $v \in \widetilde{\mathcal{W}}_{p^{\prime}}^{ \pm}$. 
Proof. Let $\varphi \in C^{1}(\mathbb{R})$ and $u \in C\left(S ; C^{1}(\bar{\Omega})\right)$. Then, for each $\omega \in S$, we have from Gauss Theorem,

$$
\int_{\Sigma_{\omega}^{+}}(\omega \cdot \nu) u \varphi(u) d \sigma=\int_{\Omega}(\omega \cdot \nabla u)\left[\varphi(u)+u \varphi^{\prime}(u)\right] d x+\int_{\Sigma_{\omega}^{-}}|\omega \cdot \nu| u \varphi(u) d \sigma .
$$

If $p \geq 2$, we consider $\varphi(s)=s|s|^{p-2}$. Then $\varphi \in C^{1}(\mathbb{R})$ and (5) takes the form

$$
\int_{\Sigma_{\omega}^{+}}(\omega \cdot \nu)|u|^{p} d \sigma=p \int_{\Omega}(\omega \cdot \nabla u) u|u|^{p-2} d x+\int_{\Sigma_{\omega}^{-}}|\omega \cdot \nu||u|^{p} d \sigma .
$$

From the Young inequality, we get

$$
\int_{\Omega}|\omega \cdot \nabla u \| u|^{p-1} d x \leq \frac{1}{p} \int_{\Omega}|\omega \cdot \nabla u|^{p} d x+\frac{1}{p^{\prime}} \int_{\Omega}|u|^{p} .
$$

By substituting (7) in (6) and integrating over $S$, we obtain (3). The general case follows by density.

For $1 \leq p<2$, we consider $\varphi_{\varepsilon}(s)=s\left(\varepsilon+s^{2}\right)^{(p-2) / 2}$. Then $\varphi \in C^{1}(\mathbb{R})$ and since

$$
\begin{aligned}
\varphi_{\varepsilon}(s) \underset{\varepsilon \rightarrow 0}{\longrightarrow} s|s|^{p-2}, & \varphi_{\varepsilon}^{\prime}(s) \underset{\varepsilon \rightarrow 0}{\longrightarrow}(p-1)|s|^{p-2}, \\
\left|\varphi_{\varepsilon}(s)\right| \leq|s|^{p-1}, & \left|\varphi_{\varepsilon}^{\prime}(s)\right| \leq(p-1)|s|^{p-2}+\varepsilon^{p / 2-1}
\end{aligned}
$$

we obtain (6) from (5) by application of Lebesgue Theorem. The conclusion follows, as before, by density.

As an immediate consequence of Lemma 2.1, we can introduce the space

$$
\widetilde{\mathcal{W}}_{p}=\left\{f \in \mathcal{W}_{p} ; \int_{\Sigma}|\omega \cdot \nu(\sigma)||f(\omega, \sigma)|^{p} d \omega d \sigma<+\infty\right\}
$$

and we have that

Corollary 2.2. $\widetilde{\mathcal{W}}_{p}^{+}=\widetilde{\mathcal{W}}_{p}^{-}=\widetilde{\mathcal{W}}_{p}$ with equivalent norms.

Lemma 2.3. The trace operators $\gamma_{ \pm}: \widetilde{\mathcal{W}}_{p}^{ \pm} \rightarrow L^{p}\left(\Sigma^{\mp} ; d \xi\right)$ are surjective. More precisely, for each $f \in L^{p}\left(\Sigma^{\mp} ; d \xi\right)$, there exists $h \in \widetilde{\mathcal{W}}_{p}^{ \pm}$such that $\gamma_{ \pm}(h)=f$ and

$$
\|h\|_{\widetilde{\mathcal{W}}_{p}^{ \pm}} \leq C\|f\|_{L^{p}\left(\Sigma^{\mp}, d \xi\right)},
$$

where $C>0$ is independent of $f$.

Proof. See $[3,4]$.

We consider the operator $A: D(A) \rightarrow L^{p}(Q)$, defined by $(A u)(\omega, x)=\omega \cdot \nabla u(\omega, x)$, with $D(A)=\left\{u \in \widetilde{\mathcal{W}}_{p} ; \gamma_{+}(u)=0\right\}$. 
Theorem 2.4. The operator $A$ is $m$-accretive in $L^{p}(Q)$, for $p \in[1,+\infty)$.

Proof. It follows from the following two lemmas.

Lemma 2.5. The operator $A$ is accretive in $L^{p}(Q)$, i.e.,

$$
\|u+\lambda A u\|_{L^{p}(Q)} \geq\|u\|_{L^{p}(Q)}, \quad \forall u \in D(A), \forall \lambda>0 .
$$

Proof. It is sufficient to prove that

$$
\|u+A u\|_{L^{p}(Q)} \geq 1, \quad \forall u \in D(A), \text { such that }\|u\|_{L^{p}(Q)}=1 .
$$

We prove first the case $1<p<\infty$. Let $u \in C\left(S ; C^{1}(\bar{\Omega})\right)$ with $u=0$ on $\Sigma^{-}$and such that $\|u\|_{L^{p}(Q)}=1$. Then

$$
\|u+A u\|_{L^{p}(Q)}=\sup \left\{\int_{Q}(u+\omega \cdot \nabla u) v d x d \omega ; v \in L^{p^{\prime}}(Q),\|v\|_{L^{p^{\prime}}(Q)}=1\right\},
$$

where $1 / p^{\prime}+1 / p=1$. If $v=|u|^{p-2} u$, then $v \in L^{p^{\prime}}(Q)$ and $\|v\|_{L^{p^{\prime}}(Q)}=1$. Hence

$$
\|u+A u\|_{L^{p}(Q)} \geq \int_{Q}\left(|u(\omega, x)|^{p}+|u(\omega, x)|^{p-2} u(\omega, x) \omega \cdot \nabla u(\omega, x)\right) d x d \omega .
$$

Since

$$
|u(\omega, x)|^{p-2} u(\omega, x) \omega \cdot \nabla u(\omega, x)=\frac{1}{p} \operatorname{div}_{x}\left(\omega|u(\omega, x)|^{p}\right),
$$

we have, from Gauss Theorem,

$$
\int_{\Omega}|u(\omega, x)|^{p-2} u(\omega, x) \omega \cdot \nabla u(\omega, x) d x=\frac{1}{p} \int_{\Sigma_{\omega}^{+}} \omega \cdot \nu(\sigma)|u(\omega, \sigma)|^{p} d \sigma \geq 0 .
$$

Therefore,

$$
\|u+A u\|_{L^{p}(Q)} \geq \int_{Q}|u(\omega, x)|^{p} d x d \omega=1 .
$$

The conclusion follows by density.

The case $p=1$ follows easily by a density argument and the fact that $\|u\|_{p} \rightarrow\|u\|_{1}$ as $p \rightarrow 1$, for all $u \in L^{2}(Q) \cap L^{1}(Q)$. (See also the Corollary 2.7 below.)

In order to prove the maximality of $A$, we consider, for $u \in L^{p}(Q)$, the extension of $u$, that is the function $\tilde{u}: S \times \mathbb{R}^{N} \rightarrow \mathbb{R}$,

$$
\tilde{u}(\omega, x)= \begin{cases}u(\omega, x) & \text { if }(\omega, x) \in S \times \Omega, \\ 0 & \text { otherwise. }\end{cases}
$$

It defines the extension operator $u \mapsto \tilde{u}$ which is a continuous operator from $L^{p}(Q)$ into $L^{p}\left(S \times \mathbb{R}^{N}\right)$. 
Let $L_{\lambda}: L^{p}(Q) \rightarrow L^{p}(Q)$ be the operator defined by

$$
\left(L_{\lambda} u\right)(\omega, x)=\int_{0}^{\infty} e^{-s} \tilde{u}(\omega, x-\lambda s \omega) d s, \quad(\omega, x) \in S \times \Omega .
$$

Lemma 2.6. $L_{\lambda}$ satisfies the following properties, for each $p \in[1+\infty)$ :

(i) $L_{\lambda} \in \mathcal{L}\left(L^{p}(Q), L^{p}(Q)\right),\left\|L_{\lambda}\right\|_{\mathcal{L}\left(L^{p}(Q), L^{p}(Q)\right)} \leq 1$;

(ii) $\forall u \in L^{p}(Q), L_{\lambda} u \underset{\lambda \rightarrow 0}{\longrightarrow} u$ in $L^{p}(Q)$;

(iii) $(I+\lambda A)\left(L_{\lambda} u\right)=u, \forall u \in L^{p}(Q)$;

(iv) $L_{\lambda} \in \mathcal{L}\left(L^{p}(Q), \mathcal{W}_{p}\right),\left\|L_{\lambda}\right\|_{\mathcal{L}\left(L^{p}(Q), \mathcal{W}_{p}\right)} \leq 2 / \lambda$;

(v) $L_{\lambda}(u) \in D(A), \forall u \in L^{p}(Q)$.

Proof. Let $u \in L^{p}(Q)$. Then,

$$
\begin{aligned}
\left|\left(L_{\lambda} u\right)(\omega, x)\right| & \leq \int_{0}^{\infty} e^{-s}|\tilde{u}(\omega, x-\lambda s \omega)| d s \\
& \leq\left(\int_{0}^{\infty} e^{-s} d s\right)^{1 / p^{\prime}}\left(\int_{0}^{\infty} e^{-s}|\tilde{u}(\omega, x-\lambda s \omega)|^{p} d s\right)^{1 / p} \\
& =\left(\int_{0}^{\infty} e^{-s}|\tilde{u}(\omega, x-\lambda s \omega)|^{p} d s\right)^{1 / p}
\end{aligned}
$$

which yields

$$
\begin{aligned}
\int_{\Omega}\left|\left(L_{\lambda} u\right)(\omega, x)\right|^{p} d x & \leq \int_{0}^{\infty} e^{-s}\left(\int_{\Omega}|\tilde{u}(\omega, x-\lambda s \omega)|^{p} d x\right) d s \\
& \leq \int_{0}^{\infty} e^{-s}\left(\int_{\mathbb{R}^{N}}|\tilde{u}(\omega, y)|^{p} d y\right) d s \\
& =\|u(\omega, \cdot)\|_{L^{p}(\Omega)}^{p} .
\end{aligned}
$$

By integrating the expression above over $S$ we obtain $\left\|L_{\lambda} u\right\|_{L^{p}(Q)} \leq\|u\|_{L^{p}(Q)}$ and the item (i) is proved. In order to prove (ii), we first note that

$$
L_{\lambda} u(\omega, x)-u(\omega, x)=\int_{0}^{\infty} e^{-s}[\tilde{u}(\omega, x-\lambda s \omega)-\tilde{u}(\omega, x)] d s .
$$

So, thanks to Hölder inequality, we have

$$
\left|L_{\lambda} u(\omega, x)-u(\omega, x)\right|^{p} \leq \int_{0}^{\infty} e^{-s}|\tilde{u}(\omega, x-\lambda s \omega)-\tilde{u}(\omega, x)|^{p} d s .
$$


By integrating this last inequality over $\Omega$ and applying Fubini's theorem, we obtain

$$
\int_{\Omega}\left|L_{\lambda} u(\omega, x)-u(\omega, x)\right|^{p} d x \leq \int_{0}^{\infty} e^{-s}\left(\int_{\mathbb{R}^{N}}|\tilde{u}(\omega, x-\lambda s \omega)-\tilde{u}(\omega, x)|^{p} d x\right) d s
$$

and the conclusion follows from the Lebesgue Theorem, since the translation operator $\left(\tau_{\lambda} g\right)(x)=g(x-\lambda s \omega)$ defines a continuous group of isometries in $L^{p}\left(\mathbb{R}^{N}\right)$, that is, for $1 \leq p<\infty$,

$$
\lim _{\lambda \rightarrow 0}\left\|\tau_{\lambda} g-g\right\|_{p}=0 .
$$

By a direct application of Fubini's Theorem, one checks easily that the adjoint of $L_{\lambda}$ is given by

$$
\left(L_{\lambda}^{*} u\right)(\omega, x)=\int_{0}^{\infty} e^{-s} \tilde{u}(\omega, x+\lambda s \omega) d s
$$

since

$$
\int_{Q}\left(L_{\lambda} u\right)(\omega, x) v(\omega, x) d x d \omega=\int_{Q}\left(L_{\lambda}^{*} v\right)(\omega, x) u(\omega, x) d x d \omega,
$$

for each $u \in L^{p}(Q)$ and $v \in L^{p^{\prime}}(Q)$. Moreover, for $u \in L^{p}(Q)$,

$$
\left\langle L_{\lambda} u(\omega, \cdot), \varphi\right\rangle=\int_{\Omega} u(\omega, x) L_{\lambda}^{*}(\varphi)(x) d x, \quad \forall \varphi \in C_{0}^{\infty}(\Omega) .
$$

On the other hand,

$$
\begin{aligned}
L_{\lambda}^{*}(\lambda A \varphi)(x) & =\lambda \int_{0}^{\infty} e^{-s} \omega \cdot \nabla \tilde{\varphi}(x+\lambda s \omega) d s \\
& =\int_{0}^{\infty} e^{-s} \frac{d}{d s} \tilde{\varphi}(x+\lambda s \omega) d s \\
& =-\varphi(x)+\left(L_{\lambda}^{*} \varphi\right)(x), \quad \forall \varphi \in C_{0}^{\infty}(\Omega)
\end{aligned}
$$

and we have

$$
\begin{aligned}
\left\langle L_{\lambda} u ; \varphi\right\rangle & =\int_{\Omega} u L_{\lambda}^{*} \varphi=\int_{\mathbb{R}^{N}} \tilde{u} L_{\lambda}^{*} \varphi=\int_{\mathbb{R}^{N}} \tilde{u}\left[\varphi+L_{\lambda}^{*}(\lambda \omega \cdot \nabla \varphi)\right] \\
& =\langle u ; \varphi\rangle+\int_{\mathbb{R}^{N}} \tilde{u} L_{\lambda}^{*}(\lambda \omega \cdot \nabla \varphi) \\
& =\langle u ; \varphi\rangle-\left\langle L_{\lambda} u ; \lambda \omega \cdot \nabla \varphi\right\rangle \\
& =\left\langle u-\lambda \omega \cdot \nabla\left(L_{\lambda} u\right) ; \varphi\right\rangle
\end{aligned}
$$

which means that $(I+\lambda A)\left(L_{\lambda} u\right)=u$ in the sense of distributions in $\Omega$.

Since $\lambda A\left(L_{\lambda} u\right)=u-L_{\lambda} u \in L^{p}(Q)$, we obtain $L_{\lambda} u \in \mathcal{W}_{p}$. Moreover,

$$
\|A u\|_{L^{p}(Q)} \leq \frac{1}{\lambda}\left(\|u\|_{L^{p}(Q)}+\left\|L_{\lambda} u\right\|_{L^{p}(Q)}\right) \leq \frac{2}{\lambda}\|u\|_{L^{p}(Q)} .
$$

In order to prove that $L_{\lambda} u \in D(A)$, we note that if $u \in C(S \times \bar{\Omega})$, then it is obvious that $u \in L^{p}(Q)$ and $L_{\lambda} u=0$ on $\Sigma^{-}$. Again, the general case follows by density. 
Corollary 2.7. Let $f \in L^{p}(Q), p \in[1,+\infty)$ and assume that $u \in D(A)$ is a solution of $u+A u=f$. If $f \geq 0$ a.e. in $Q$, then $u \geq 0$ a.e. in $Q$. In particular, it follows that

$$
\|u\|_{L^{1}(Q)} \leq\|f\|_{L^{1}(Q)} .
$$

Proof. Since $\left(L_{1} f\right)(\omega, x)=\int_{0}^{\infty} e^{-s} \tilde{f}(\omega, x-s \omega) d s \geq 0$ if $f \geq 0$, we obtain $u \geq 0$ from items (iii) and (v) in Lemma 2.6.

Now assume that $f \in L^{1}(Q)$ and let $v \in D(A)$ the solution of $v+A v=|f|$. Then $v \geq 0$ and $-|f| \leq f \leq|f|$ implies that $|u| \leq v$. Therefore, by integrating over $Q$, we obtain

$$
\|u\|_{L^{1}(Q)} \leq \int_{Q} v(\omega, x) d x d \omega \leq \int_{Q}[v(\omega, x)+A v(\omega, x)] d x d \omega=\|f\|_{L^{1}(Q)} .
$$

It follows from Theorem 2.4 and Corollary 2.7 that the operator $A$ generates a positive semigroup $\left\{U_{0}(t)\right\}_{t \geq 0}$ of contractions acting on $L^{p}(Q)$.

Let $q \in L^{\infty}(\Omega)$ and $f: \Omega \times S \times S \rightarrow \mathbb{R}$ be a real measurable function satisfying

$$
\begin{cases}\int_{S}\left|f\left(x, \omega^{\prime}, \omega\right)\right| d \omega^{\prime} \leq M_{1} & \text { a.e. } \Omega \times S, \\ \int_{S}\left|f\left(x, \omega^{\prime}, \omega\right)\right| d \omega \leq M_{2} & \text { a.e. } \Omega \times S .\end{cases}
$$

Associated to these functions, we define the following continuous operators:

(a) $B \in \mathcal{L}\left(L^{p}(Q), L^{p}(Q)\right)$ defined by $B[u](\omega, x)=q(x) u(\omega, x)$,

(b) $K_{f}[u](\omega, x)=\int_{S} f\left(x, \omega^{\prime}, \omega\right) u\left(\omega^{\prime}, x\right) d \omega^{\prime}$.

It follows from (8) that $K_{f} \in \mathcal{L}\left(L^{p}(Q), L^{p}(Q)\right) \forall p \in[1,+\infty)$ and (see [7])

$$
\left\|K_{f}[u]\right\|_{L^{p}(Q)} \leq M_{1}^{1 / p^{\prime}} M_{2}^{1 / p}\|u\|_{L^{p}(Q)} .
$$

The operator $A+B-K_{f}: D(A) \rightarrow L^{p}(Q)$ generates a $c_{0}$-semigroup $\{U(t)\}_{t \geq 0}$ on $L^{p}(Q)$ satisfying

$$
\|U(t)\|_{\mathcal{L}} \leq e^{C t}, \quad C=\left\|q^{-}\right\|_{\infty}+M_{2}
$$

\section{Stability and identification of parameters}

We consider the initial-boundary value problem for the linear Boltzmann equation

$$
\left\{\begin{array}{l}
\partial_{t} u(t, \omega, x)+\omega \cdot \nabla u(t, \omega, x)+q(x) u(t, \omega, x)=K_{f}[u](t, \omega, x) \\
u(0, \omega, x)=0, \quad(\omega, x) \in S \times \Omega \\
u(t, \omega, \sigma)=\varphi(t, \omega, \sigma), \quad(\omega, \sigma) \in \Sigma^{-}, \quad t \in(0, T)
\end{array}\right.
$$

where $q \in L^{\infty}(\Omega)$ and

$$
K_{f}[u](t, \omega, x)=\int_{S} f\left(x, \omega^{\prime}, \omega\right) u\left(t, \omega^{\prime}, x\right) d \omega^{\prime},
$$


with $f$ satisfying (8).

By the results stated in section 2 , it follows that, for any $p \in[1,+\infty)$, if $\varphi \in L^{p}\left(0, T ; L^{p}\left(\Sigma^{-}, d \xi\right)\right)$, there exists a unique solution $u \in C\left([0, T] ; \widetilde{\mathcal{W}}_{p}\right) \cap$ $C^{1}\left([0, T] ; L^{p}(Q)\right)$ of $(9)$. This solution $u$ allows us to define the albedo operator

$$
\begin{gathered}
\mathcal{A}_{q, f}: L^{p}\left(0, T ; L^{p}\left(\Sigma^{-}, d \xi\right)\right) \rightarrow L^{p}\left(0, T ; L^{p}\left(\Sigma^{+}, d \xi\right)\right) \\
\mathcal{A}_{q, f}[\varphi](t, \omega, \sigma)=u(t, \omega, \sigma), \quad(\omega, \sigma) \in \Sigma^{+} .
\end{gathered}
$$

As a consequence of Lemmas 2.1 and $2.3, \mathcal{A}_{q, f}$ is a linear and bounded operator. So, we denote by $\left\|\mathcal{A}_{q, f}\right\|_{p}$ its norm. In order to simplify our notation, we will consider from now on $\|\cdot\|_{L_{p}^{ \pm}}=\|\cdot\|_{L^{p}\left(0, T ; L^{p}\left(\Sigma^{ \pm}, d \xi\right)\right)}$.

We also consider the following backward-boundary value problem, called the $a d$ joint problem of (9):

$$
\left\{\begin{array}{l}
\partial_{t} v(t, \omega, x)+\omega \cdot \nabla v(t, \omega, x)-q(x) v(t, \omega, x)=-K_{f}^{*}[v](t, \omega, x) \\
v(T, \omega, x)=0, \quad(\omega, x) \in S \times \Omega \\
v(t, \omega, \sigma)=\psi(t, \omega, \sigma), \quad(\omega, \sigma) \in \Sigma^{+}, t \in(0, T)
\end{array}\right.
$$

where $\psi \in L^{p^{\prime}}\left(0, T ; L^{p^{\prime}}\left(\Sigma^{+}, d \xi\right)\right), p^{\prime} \in[1,+\infty)$,

$$
K_{f}^{*}[v]\left(t, \omega^{\prime}, x\right)=\int_{S} f\left(x, \omega^{\prime}, \omega\right) v(t, \omega, x) d \omega
$$

with the corresponding albedo operator $\mathcal{A}_{q, f}^{*}$

$$
\begin{gathered}
\mathcal{A}_{q, f}^{*}: L^{p^{\prime}}\left(0, T ; L^{p^{\prime}}\left(\Sigma^{+}, d \xi\right)\right) \rightarrow L^{p^{\prime}}\left(0, T ; L^{p^{\prime}}\left(\Sigma^{-}, d \xi\right)\right) \\
\mathcal{A}_{q, f}^{*}[\psi](t, \omega, \sigma)=v(t, \omega, \sigma), \quad(\omega, \sigma) \in \Sigma^{-} .
\end{gathered}
$$

The operators $\mathcal{A}_{q, f}$ and $\mathcal{A}_{q, f}^{*}$ satisfy the following property:

Lemma 3.1. Let $\varphi \in L^{p}\left(0, T ; L^{p}\left(\Sigma^{-} ; d \xi\right)\right)$ and $\psi \in L^{p^{\prime}}\left(0, T ; L^{p^{\prime}}\left(\Sigma^{+} ; d \xi\right)\right)$, where $p, p^{\prime} \in(1,+\infty)$ are such that $1 / p+1 / p^{\prime}=1$. Then, we have

$$
\begin{aligned}
\int_{0}^{T} \int_{\Sigma^{-}}(\omega \cdot \nu(\sigma)) \varphi(t, \omega, \sigma) \mathcal{A}_{q, f}^{*}[\psi](t, \omega, \sigma) d \sigma d \omega d t= \\
\quad=-\int_{0}^{T} \int_{\Sigma^{+}}(\omega \cdot \nu(\sigma)) \psi(t, \omega, \sigma) \mathcal{A}_{q, f}[\varphi](t, \omega, \sigma) d \sigma d \omega d t .
\end{aligned}
$$

Proof. It is a direct consequence of Lemma 2.1. Let $u(t, \omega, x)$ the solution of (9) with boundary condition $\varphi$ and $v(t, \omega, x)$ the solution of (10) with boundary condition $\psi$. We obtain the result by using (4), once the equation in (9) is multiplied by $v$ and integrated over $Q$. 
Lemma 3.2. Let $T>0, q_{1}, q_{2} \in L^{\infty}(\Omega)$ and $f_{1}, f_{2}$ satisfying (8). Assume that $u_{1}$ is the solution of $(9)$ with $(q, f)=\left(q_{1}, f_{1}\right)$ and boundary condition $\varphi \in L^{p}(0, T$; $\left.L^{p}\left(\Sigma^{-}, d \xi\right)\right), p \in(1,+\infty)$ and that $v_{2}$ is the solution of $(10)$, with $(q, f)=\left(q_{2}, f_{2}\right)$ and boundary condition $\psi \in L^{p^{\prime}}\left(0, T ; L^{p^{\prime}}\left(\Sigma^{+}, d \xi\right)\right), 1 / p+1 / p^{\prime}=1$. Then we have

$$
\begin{aligned}
\int_{0}^{T} \int_{Q}\left(q_{2}(x)-q_{1}(x)\right) & u_{1}(t, \omega, x) v_{2}(t, \omega, x) d x d \omega d t \\
+ & \int_{0}^{T} \int_{Q} K_{f_{1}-f_{2}}\left[u_{1}\right](t, \omega, x) v_{2}(t, \omega, x) d x d \omega d t \\
& =\int_{0}^{T} \int_{\Sigma^{+}}(\omega \cdot \nu(\sigma))\left[\mathcal{A}_{1}[\varphi]-\mathcal{A}_{2}[\varphi]\right](t, \omega, \sigma) \psi(t, \omega, \sigma) d \sigma d \omega d t
\end{aligned}
$$

where $\mathcal{A}_{j}=\mathcal{A}_{q_{j}, f_{j}}, j=1,2$.

Proof. It is a direct consequence of Lemma 3.1.

Lemma 3.3. Let $T>0, q_{1}, q_{2} \in L^{\infty}(\Omega)$, and $f_{1}, f_{2}$ satisfying (8). We consider $\psi_{1}, \psi_{2} \in C\left(S, C_{0}^{\infty}\left(\mathbb{R}^{N}\right)\right)$ such that

$$
\operatorname{supp} \psi_{1}(\omega, \cdot) \cap \bar{\Omega}=\left(\operatorname{supp} \psi_{2}(\omega, \cdot)+T \omega\right) \cap \bar{\Omega}=\emptyset, \quad \forall \omega \in S .
$$

Then, there exists $C_{0}>0$ such that, for each $\lambda>0$, there exist $R_{1, \lambda} \in C\left([0, T] ; \widetilde{\mathcal{W}}_{p}\right)$ and $R_{2, \lambda}^{*} \in C\left([0, T] ; \widetilde{\mathcal{W}}_{p^{\prime}}\right)$ satisfying

$$
\left\|R_{1, \lambda}\right\|_{C\left([0, T] ; L^{p}(Q)\right)} \leq C_{0}, \quad\left\|R_{2, \lambda}^{*}\right\|_{C\left([0, T] ; L^{p^{\prime}}(Q)\right)} \leq C_{0}, \quad \forall p, p^{\prime} \in(1,+\infty),
$$

with $1 / p+1 / p^{\prime}=1$, for which the functions $u_{1}, v_{2}$ defined by

$$
\left\{\begin{aligned}
u_{1}(t, \omega, x)= & \psi_{1}(\omega, x-t \omega) \exp \left(-\int_{0}^{t} \widetilde{q}_{1}(x-s \omega) d s+\mathrm{i} \lambda(t-\omega \cdot x)\right) \\
& +R_{1, \lambda}(t, \omega, x) \\
v_{2}(t, \omega, x)= & \psi_{2}(\omega, x-t \omega) \exp \left(\int_{0}^{t} \widetilde{q}_{2}(x-s \omega) d s-\mathrm{i} \lambda(t-\omega \cdot x)\right) \\
& +R_{2, \lambda}^{*}(t, \omega, x)
\end{aligned}\right.
$$

are solutions of $(9)$ with $(q, f)=\left(q_{1}, f_{1}\right)$ and $(10)$ with $(q, f)=\left(q_{2}, f_{2}\right)$ respectively. Moreover, if $f_{1}, f_{2} \in L^{\infty}\left(\Omega ; L^{2}(S \times S)\right)$, then we have

$$
\lim _{\lambda \rightarrow+\infty}\left\|R_{1, \lambda}\right\|_{L^{2}([0, T] \times Q)}=\lim _{\lambda \rightarrow+\infty}\left\|R_{2, \lambda}^{*}\right\|_{L^{2}([0, T] \times Q)}=0 .
$$

Proof. Let $u$ be the function

$$
u(t, \omega, x)=\psi_{1}(\omega, x-t \omega) \exp \left(-\int_{0}^{t} \tilde{q}_{1}(x-s \omega) d s+\mathrm{i} \lambda(t-\omega \cdot x)\right)+R(t, \omega, x) .
$$


By direct calculations, we easily verify that

$$
\partial_{t} u+\omega \cdot \nabla u+q_{1} u-K_{f_{1}}[u]=\partial_{t} R+\omega \cdot \nabla R+q_{1} R-K_{f_{1}}[R]-\exp (\mathrm{i} \lambda t) z_{1, \lambda},
$$

where

$$
z_{1, \lambda}(t, \omega, x)=\int_{S} f_{1}\left(x, \omega^{\prime}, \omega\right) \psi_{1}\left(\omega^{\prime}, x-t \omega^{\prime}\right) e^{-\int_{0}^{t} \tilde{q}_{1}\left(x-s \omega^{\prime}\right) d s-\mathrm{i} \lambda \omega^{\prime} \cdot x} d \omega^{\prime} .
$$

By choosing $R_{1, \lambda} \in C^{1}\left([0, T] ; L^{p}(Q)\right) \cap C([0, T] ; D(A))$ the solution of

$$
\begin{cases}\partial_{t} R+\omega \cdot \nabla R+q_{1} R & =K_{f_{1}}[R]+\exp (\mathrm{i} \lambda t) z_{1, \lambda}, \\ R(0, \omega, x) & =0, \quad(\omega, x) \in S \times \Omega, \\ R(t, \omega, \sigma) & =0, \quad(\omega, \sigma) \in \Sigma^{-},\end{cases}
$$

we see that (11) implies that the function $u$ defined by (15) satisfies (9) with boundary condition

$$
\varphi(t, \omega, \sigma)=\psi_{1}(\omega, \sigma-t \omega) e^{-\int_{0}^{t} \tilde{q}_{1}(\sigma-s \omega) d s+\mathrm{i} \lambda(t-\omega \cdot \sigma)}, \quad(\omega, \sigma) \in \Sigma^{-} .
$$

Multiplying both sides of the equation in (16) by the complex conjugate of $|R|^{p-2} R$, integrating it over $Q$ and taking its real part, we get, from Lemma 2.1,

$$
\begin{aligned}
& \frac{1}{p} \frac{d}{d t} \int_{Q}|R(t)|^{p} d \omega d x+\frac{1}{p} \int_{\Sigma^{+}} \omega \cdot \nu(\sigma)|R(t)|^{p} d \omega d \sigma+\int_{Q} q_{1}|R(t)|^{p} d \omega d x \\
& \quad-\Re \int_{Q} K_{f_{1}}[R(t)]|R(t)|^{p-2} \overline{R(t)} d \omega d x=\Re\left[e^{\mathrm{i} \lambda t} \int_{Q} z_{1, \lambda}(t)|R(t)|^{p-2} \overline{R(t)} d \omega d x\right] .
\end{aligned}
$$

It follows from the Hölder inequality and (8) that

$$
\int_{Q}\left|K_{f_{1}}[R(t)]\right||R(t)|^{p-1} d x d \omega \leq C_{p}\|R(t)\|_{L^{p}(Q)}^{p},
$$

where $C_{p}=M_{1}^{1 / p^{\prime}} M_{2}^{1 / p} \leq \max \left\{M_{1}, M_{2}\right\}$. Therefore, considering the decomposition $q_{1}=q_{1}^{+}-q_{1}^{-}$, we obtain,

$$
\frac{d}{d t}\|R(t)\|_{L^{p}(Q)}^{p} \leq p C_{1}\|R(t)\|_{L^{p}(Q)}^{p}+\left\|z_{1, \lambda}(t)\right\|_{L^{p}(Q)}^{p},
$$

where $C_{1}=\left\|q_{1}^{-}\right\|_{\infty}+\max \left\{M_{1}, M_{2}\right\}+1$ and the Gronwall inequality implies that

$$
\|R(t)\|_{L^{p}(Q)}^{p} \leq\left\|z_{1, \lambda}(t)\right\|_{L^{p}(Q)}^{p} e^{p T C_{1}}, \quad \forall t \in[0, T] .
$$

The first inequality in (12) follows easily because

$$
\left|z_{1, \lambda}(t, \omega, x)\right| \leq\left\|\psi_{1}\right\|_{\infty} e^{\left\|q_{1}^{-}\right\|_{\infty} T} M_{1} .
$$


Since the same arguments hold for $v_{2}$ and $R_{2, \lambda}^{*}$ with $p^{\prime}$ in place of $p$, we obtain the second inequality in (12).

We assume now $f \in L^{\infty}\left(\Omega ; L^{2}(S \times S)\right)$. Following the same steps as before, we obtain

$$
\|R(t)\|_{L^{2}(Q)}^{2} \leq\left\|z_{1, \lambda}(t)\right\|_{L^{2}(Q)}^{2} e^{2 C_{1} T}, \quad \forall t \in[0, T],
$$

where $C_{1}=\left\|q_{1}^{-}\right\|_{\infty}+\|f\|_{L^{\infty}\left(\Omega ; L^{2}(S \times S)\right)}+1$. Since for each $x \in \mathbb{R}^{N}$, the map $\omega \mapsto \exp (\mathrm{i} \lambda \omega \cdot x)$ converges weakly to zero in $L^{2}(S)$ when $\lambda \rightarrow+\infty$ and the integral operator with kernel $f_{1}(x, \cdot, \cdot)$ is compact in $L^{2}(S)$, we obtain

$$
\lim _{\lambda \rightarrow+\infty}\left\|z_{1, \lambda}(t, \cdot, x)\right\|_{L^{2}(S)}=0 \quad \text { a.e. in } \quad[0, T] \times \Omega .
$$

Moreover, $\left\|z_{1, \lambda}(t, \cdot, x)\right\|_{L^{2}(S)} \leq C$, where $C>0$ is a constant that does not depend on $\lambda$. The Lebesgue's Dominated Convergence Theorem (Lebesgue Theorem in short) implies that

$$
\lim _{\lambda \rightarrow+\infty}\left\|z_{1, \lambda}\right\|_{L^{2}([0, T] \times Q)}=0 .
$$

From (18) and (17) we obtain (14), and our proof is complete.

We are now in position to prove our main result.

Proof of Theorem 1.1. Let $\varepsilon=(T-\operatorname{diam}(\Omega)) / 2$ and consider

$$
\Omega_{\varepsilon}=\left\{x \in \mathbb{R}^{N} \backslash \bar{\Omega} ; \operatorname{dist}(x, \Omega)<\varepsilon\right\} .
$$

Let $\chi \in C(S)$ and $\Phi, \Psi \in C_{0}^{\infty}\left(\Omega_{\varepsilon}\right)$. We define $\psi_{1}(\omega, x)=\chi(\omega) \Phi(x)$ and $\psi_{2}(\omega, x)=$ $\Psi(x)$. Since $T>\operatorname{diam}(\Omega)$ implies that $\psi_{1}, \psi_{2}$ satisfy (11), we may consider the solutions $u_{1}$ and $v_{2}$ defined by (13). Denoting by $f=f_{1}-f_{2}$ and $\rho=\tilde{q}_{1}-\tilde{q}_{2}\left(\tilde{q}_{j}\right.$ being the zero extension of $q_{j}$ in the exterior of $\Omega$ ), we have by Lemma 3.2,

$$
\begin{aligned}
& \mid \int_{0}^{T} \int_{Q} \rho(x) e^{-\int_{0}^{t} \rho(x-s \omega) d s} \chi(\omega) \Phi(x-t \omega) \Psi(x-t \omega) \\
& \quad+\int_{0}^{T} \int_{Q} z_{\lambda}(t, \omega, x) \Psi(x-t \omega) e^{\int_{0}^{t} \tilde{q}_{2}(x-s \omega) d s+\mathrm{i} \lambda \omega \cdot x} d x d \omega d t+I_{\lambda} \mid \\
& \leq\left\|\mathcal{A}_{1}-\mathcal{A}_{2}\right\|\|\varphi\|_{L_{p}^{-}}\|\psi\|_{L_{p^{\prime}}^{+}},
\end{aligned}
$$

where

$$
\left\{\begin{array}{l}
z_{\lambda}(t, \omega, x)=\int_{S} f\left(x, \omega^{\prime}, \omega\right) \chi\left(\omega^{\prime}\right) \Phi\left(x-t \omega^{\prime}\right) e^{-\mathrm{i} \lambda \omega^{\prime} \cdot x-\int_{0}^{t} \tilde{q}_{1}\left(x-s \omega^{\prime}\right) d s} d \omega^{\prime} \\
\varphi(t, \omega, \sigma)=\chi(\omega) \Phi(\sigma-t \omega) e^{\mathrm{i} \lambda(t-\omega \cdot \sigma)-\int_{0}^{t} \tilde{q}_{1}(\sigma-s \omega) d s} \\
\psi(t, \omega, \sigma)=\Psi(\sigma-t \omega) e^{\int_{0}^{t} \tilde{q}_{2}(\sigma-s \omega) d s-\mathrm{i} \lambda(t-\omega \cdot \sigma)}
\end{array}\right.
$$

and $I_{\lambda}$ represents the sum of the integrals that contain terms with $R_{1, \lambda}$ and $R_{2, \lambda}^{*}$. 
Because $R_{1, \lambda}, R_{2, \lambda}^{*}$ and $z_{\lambda}$ converge to zero in $L^{2}([0, T] \times Q)$, we get, after taking the limit as $\lambda \rightarrow+\infty$,

$$
\begin{aligned}
&\left|\int_{0}^{T} \int_{S \times \mathbb{R}^{N}} \rho(x) e^{-\int_{0}^{t} \rho(x-s \omega) d s} \chi(\omega) \Phi(x-t \omega) \Psi(x-t \omega) d x d \omega d t\right| \\
& \leq\left\|\mathcal{A}_{1}-\mathcal{A}_{2}\right\|\left\|_{p}\right\| \varphi\left\|_{L_{p}^{-}}\right\| \psi \|_{L_{p^{\prime}}^{+}}
\end{aligned}
$$

Since

$$
\begin{aligned}
\int_{0}^{T} \int_{S \times \mathbb{R}^{N}} \rho(x) e^{-\int_{0}^{t} \rho(x-s \omega) d s} \chi(\omega) \Phi(x-t \omega) \Psi(x-t \omega) d x d \omega d t \\
=\int_{0}^{T} \int_{S \times \mathbb{R}^{N}} \rho(y+t \omega) e^{-\int_{0}^{t} \rho(y+s \omega) d s} \chi(\omega) \Phi(y) \Psi(y) d y d \omega d t \\
=\int_{S \times \mathbb{R}^{N}}\left[1-e^{-\int_{0}^{T} \rho(y+s \omega) d s}\right] \chi(\omega) \Phi(y) \Psi(y) d y d \omega,
\end{aligned}
$$

it follows that

$$
\left|\int_{S \times \mathbb{R}^{N}}\left[1-e^{-\int_{0}^{T} \rho(y+s \omega) d s}\right] \chi(\omega) \Phi(y) \Psi(y) d y d \omega\right| \leq\left\|\mathcal{A}_{1}-\mathcal{A}_{2}\right\|\left\|_{p}\right\|\left\|_{L_{p}^{-}}\right\| \psi \|_{L_{p^{\prime}}^{+}} .
$$

Note that

$$
\left\{\begin{array}{l}
\|\psi\|_{L_{p^{\prime}}^{+}} \leq C_{1}\|\Psi\|_{L^{\infty}\left(\mathbb{R}^{N}\right)}, \quad C_{1}=C(T, M,|S|,|\partial \Omega|) \\
\lim _{p \rightarrow 1}\|\varphi\|_{L_{p}^{-}}=\|\varphi\|_{L_{1}^{-}} \\
\limsup _{p \rightarrow 1}\left\|\mathcal{A}_{1}-\mathcal{A}_{2}\right\|_{p} \leq\left\|\mathcal{A}_{1}-\mathcal{A}_{2}\right\|_{1}
\end{array}\right.
$$

which yields

$$
\left|\int_{S \times \mathbb{R}^{N}}\left[1-e^{-\int_{0}^{T} \rho(y+s \omega) d s}\right] \chi(\omega) \Phi(y) \Psi(y) d y d \omega\right| \leq C_{1}\left\|\mathcal{A}_{1}-\mathcal{A}_{2}\right\|_{1}\|\varphi\|_{L_{1}^{-}}\|\Psi\|_{L^{\infty}} .
$$

Note also that $\|\varphi\|_{L_{1}^{-}} \leq C_{2}\|\Phi\|_{L^{1}\left(\mathbb{R}^{N}\right)}\|\chi\|_{L^{1}(S)}$, for some $C_{2}$ that depends as $C_{1}$ on $T, M,|S|$, and $|\partial \Omega|$. Hence,

$$
\begin{aligned}
\left|\int_{S \times \mathbb{R}^{N}}\left[1-e^{-\int_{0}^{T} \rho(y+s \omega) d s}\right] \chi(\omega) \Phi(y) \Psi(y) d y d \omega\right| \\
\leq C_{3}\left\|\mathcal{A}_{1}-\mathcal{A}_{2}\right\|_{1}\|\Phi\|_{L^{1}\left(\mathbb{R}^{N}\right)}\|\Psi\|_{L^{\infty}\left(\mathbb{R}^{N}\right)}\|\chi\|_{L^{1}(S)} .
\end{aligned}
$$

Let $\mathcal{O} \subset \Omega_{\varepsilon}$ be an open set such that $\operatorname{supp} \Phi, \operatorname{supp} \Psi \subset \mathcal{O}$. Assume that $\Psi \leq 1$. Taking a sequence $\left\{\Psi_{k}\right\}_{k}$ such that $\Psi_{k}$ converges a.e. in $\mathbb{R}^{N}$ to $\mathbf{1}_{\mathcal{O}}$, the characteristic function of $\mathcal{O}$, we obtain from Lebesgue Theorem

$$
\left|\int_{S \times \mathcal{O}}\left[1-e^{-\int_{0}^{T} \rho(y+s \omega) d s}\right] \chi(\omega) \Phi(y) d y d \omega\right| \leq C_{3}\left\|\mathcal{A}_{1}-\mathcal{A}_{2}\right\|_{1}\|\Phi\|_{L^{1}\left(\mathbb{R}^{N}\right)}\|\chi\|_{L^{1}(S)} .
$$


Now, taking the supremum of the above inequality among all $\Phi$ with $\|\Phi\|_{L^{1}\left(\mathbb{R}^{N}\right)}=1$, we get

$$
\left\|\int_{S}\left[1-e^{-\int_{0}^{T} \rho(y+s \omega) d s}\right] \chi(\omega) d \omega\right\|_{L^{\infty}(\mathcal{O})} \leq C_{3}\left\|\mathcal{A}_{1}-\mathcal{A}_{2}\right\|_{1}\|\chi\|_{L^{1}(S)} .
$$

In particular,

$$
\left|\int_{S}\left[1-e^{-\int_{0}^{T} \rho(y+s \omega) d s}\right] \chi(\omega) d \omega\right| \leq C_{3}\left\|\mathcal{A}_{1}-\mathcal{A}_{2}\right\|_{1}\|\chi\|_{L^{1}(S)},
$$

for a.e. $y \in \mathcal{O}$. Since $\mathcal{O}$ can be chosen arbitrarily in $\Omega_{\varepsilon}$, we obtain (19) for a.e. $y \in \Omega_{\varepsilon}$. Again, taking the supremum among all $\chi \in C(S)$, with $\|\chi\|_{L^{1}(S)}=1$, we get

$$
\left|e^{-\int_{0}^{T} \rho(y+s \omega) d s}-1\right| \leq C_{3}\left\|\mathcal{A}_{1}-\mathcal{A}_{2}\right\|_{1}
$$

for a.e. $(\omega, y) \in S \times \Omega_{\varepsilon}$.

Since $\left\|q_{j}\right\|_{\infty} \leq M, j=1,2$, the Mean Value Theorem gives

$$
\left|e^{-\int_{0}^{T} \rho(y+s \omega) d s}-1\right| \geq e^{-2 T M}\left|\int_{0}^{T} \rho(y+s \omega) d s\right| .
$$

and consequently

$$
\left|\int_{0}^{T} \rho(y+s \omega) d s\right| \leq e^{2 T M} C_{3}\left\|\mathcal{A}_{1}-\mathcal{A}_{2}\right\|_{1}
$$

for a.e. $(\omega, y) \in S \times \Omega_{\varepsilon}$. Since $T>\operatorname{diam}(\Omega)$ and $\operatorname{supp} \rho \subset \Omega$, we obtain

$$
\left|\int_{-\infty}^{\infty} \rho(y+s \omega) d s\right| \leq e^{2 T M} C_{3}\left\|\mathcal{A}_{1}-\mathcal{A}_{2}\right\|_{1}
$$

for a.e. $(\omega, y) \in S \times \mathbb{R}^{N}$.

Since $\Omega$ is bounded, there exists a $R>0$ such that $\Omega \subset B_{R}$, so that we can rewrite (20) as

$$
|P[\rho](\omega, y)| \leq C_{4}\left\|\mathcal{A}_{1}-\mathcal{A}_{2}\right\|_{1}
$$

for a.e. $\omega \in S$ and a.e. $y \in \omega^{\perp} \cap B_{R}$, where $P[\rho]$ denotes the X-ray transform of $\rho$. Taking the square on both sides of (21), we obtain

$$
\|P[\rho]\|_{L^{2}(\mathcal{T})}^{2}=\int_{S} \int_{\omega^{\perp} \cap B_{R}}|P[\rho](\omega, y)|^{2} d y d \omega \leq C_{5}\left\|\mathcal{A}_{1}-\mathcal{A}_{2}\right\|_{1}^{2},
$$

where $\mathcal{T}=\left\{(\omega, y) ; \omega \in S, y \in \omega^{\perp}\right\}$ denotes the tangent bundle. 
For the X-ray transform, we have the following well known estimate (see [10])

$$
\|\rho\|_{H^{-1 / 2}} \leq C\|P[\rho]\|_{L^{2}(\mathcal{T})},
$$

where $C>0$ depends only on $N$. Combining (22) and (23) we obtain (2). The conclusion follows from interpolation formulæ and classical Sobolev imbedding theorems.

Proof of Theorem 1.2. Since we are assuming that $\mathcal{A}_{q_{1}, f_{1}}=\mathcal{A}_{q_{2}, f_{2}}, q_{1}=q_{2}$ follows trivially from (2). As a consequence, the identity in Lemma 3.2 is reduced to

$$
\int_{0}^{T} \int_{Q} K_{f_{1}-f_{2}}\left[u_{1}\right](t, \omega, x) v_{2}(t, \omega, x) d x d \omega d t=0 .
$$

Assuming that $f_{j}\left(x, \omega^{\prime}, \omega\right)=g_{j}(x) h\left(\omega^{\prime}, \omega\right)$, where $h \in L^{\infty}(S \times S)$ and $h(\omega, \omega) \neq 0$ a.e. on $S$, we have that $f_{j}$ satisfies (8) with $M_{1}=M_{2}=\alpha_{N}\left\|g_{j}\right\|_{\infty}\|h\|_{\infty}$, where $\alpha_{N}=2 \pi^{N / 2} / \Gamma(N / 2)$ is the measure of the unit sphere of $\mathbb{R}^{N}$.

For $0<r<1$ we define $\chi_{r}: S \times S \rightarrow \mathbb{R}$ as $\chi_{r}\left(\omega, \omega^{\prime}\right)=P\left(r \omega, \omega^{\prime}\right)$, where $P$ is the Poisson kernel for $B_{1}(0)$, i.e.,

$$
P(x, y)=\frac{1-|x|^{2}}{\alpha_{N}|x-y|^{N}} .
$$

From the well known properties of $P$ (see Theorem 2.46 of [8]), we have

$$
\begin{gathered}
0 \leq \chi_{r}\left(\omega, \omega^{\prime}\right) \leq \frac{2}{\alpha_{N}(1-r)^{N-1}}, \quad \forall \omega, \omega^{\prime} \in S, \\
\int_{S} \chi_{r}\left(\omega, \omega^{\prime}\right) d \omega^{\prime}=1, \quad \forall 0<r<1, \quad \forall \omega \in S \\
\lim _{r \rightarrow 1} \int_{S} \chi_{r}\left(\omega, \omega^{\prime}\right) \psi\left(\omega^{\prime}\right) d \omega^{\prime}=\psi(\omega),
\end{gathered}
$$

where the limit is taken in the topology of $L^{p}(S), p \in[1,+\infty)$ and uniformly on $S$ if $\psi \in C(S)$.

As before, we take $\Omega_{\varepsilon}=\left\{x \in \mathbb{R}^{N} \backslash \Omega\right.$; $\left.\operatorname{dist}(x, \Omega)<\varepsilon\right\}$, where $\varepsilon=(T-\operatorname{diam}(\Omega)) / 2$. For $\tilde{\omega} \in S, 0<r, r^{\prime}<1$, we define the functions $\psi_{1}(\omega, x)=\chi_{r}(\tilde{\omega}, \omega) \Phi(x)$ and $\psi_{2}(\omega, x)=\chi_{r^{\prime}}(\tilde{\omega}, \omega) \Psi(x)$, where $\Phi, \Psi \in C_{0}^{\infty}\left(\Omega_{\varepsilon}\right)$,

It follows from Lemma 3.3 that there exist functions $u_{1, r}$ and $v_{2, r^{\prime}}$ in such way that (24) takes the form

$$
\begin{aligned}
\int_{0}^{T} \int_{Q}\left[\eta(x) \int_{S} h\left(\omega, \omega^{\prime}\right) \chi_{r}\left(\tilde{\omega}, \omega^{\prime}\right) \Phi\left(x-t \omega^{\prime}\right) e^{-\int_{0}^{t} \tilde{q}\left(x-s \omega^{\prime}\right) d s-\mathrm{i} \lambda x \cdot \omega^{\prime}} d \omega^{\prime}\right] \\
\times \chi_{r^{\prime}}(\tilde{\omega}, \omega) \Psi(x-t \omega) e^{\int_{0}^{t} \tilde{q}(x-s \omega) d s+\mathrm{i} \lambda x \cdot \omega} d x d \omega d t=I_{\lambda, r^{\prime}, r}(\tilde{\omega}),
\end{aligned}
$$


where $\tilde{q}=\tilde{q}_{1}=\tilde{q}_{2}, \eta=\tilde{g}_{1}-\tilde{g}_{2}$ and $I_{\lambda, r, r^{\prime}}$ denotes the sum of the integrals that contain the terms with $R_{1, \lambda, r}$ and $R_{2, \lambda, r^{\prime}}^{*}$.

First of all, we write the left hand side of $(25)$ as

$$
J_{\lambda, r^{\prime}, r}(\tilde{\omega})=\int_{0}^{T} \int_{Q} \eta K_{h}\left[\chi_{r}(\tilde{\omega}, \cdot) u_{0, \lambda}\right] \chi_{r^{\prime}}(\tilde{\omega}, \cdot) v_{0, \lambda} d x d \omega d t,
$$

where

$$
\left\{\begin{array}{l}
u_{0, \lambda}(t, \omega, x)=\Phi(x-t \omega) \exp \left[-\int_{0}^{t} \tilde{q}(x-s \omega) d s-\mathrm{i} \lambda \omega \cdot x\right] \\
v_{0, \lambda}(t, \omega, x)=\Psi(x-t \omega) \exp \left[\int_{0}^{t} \tilde{q}(x-s \omega) d s+\mathrm{i} \lambda \omega \cdot x\right] .
\end{array}\right.
$$

Taking into account the properties of $\chi_{r}$, we have, as $r \rightarrow 1$,

$$
K_{h}\left[\chi_{r}(\tilde{\omega}, \cdot) u_{0, \lambda}\right](t, \omega, x) \rightarrow h(\omega, \tilde{\omega}) u_{0, \lambda}(t, \tilde{\omega}, x)
$$

a.e. in $[0, T] \times S \times Q$. Moreover, since $\int_{S} \chi_{r}\left(\tilde{\omega}, \omega^{\prime}\right) d \omega^{\prime}=1$ for $0<r<1$, it follows that

$$
\left|\eta K_{h}\left[\chi_{r}(\tilde{\omega}, \cdot) u_{0, \lambda}\right] \chi_{r^{\prime}}(\tilde{\omega}, \cdot) v_{0, \lambda}\right| \leq C_{N}\left(1-r^{\prime}\right)^{1-N},
$$

a.e. in $[0, T] \times Q$, where $C_{N}>0$ is independent of $r$. Therefore, as an application of Lebesgue Theorem, we have

$$
J_{\lambda, r^{\prime}, r}(\tilde{\omega}) \rightarrow J_{\lambda, r^{\prime}}(\tilde{\omega}), \quad \text { a.e. } \tilde{\omega} \in S,
$$

where

$$
\begin{aligned}
J_{\lambda, r^{\prime}}(\tilde{\omega}) & =\int_{0}^{T} \int_{Q} \eta(x) h(\tilde{\omega}, \omega) u_{0, \lambda}(t, \tilde{\omega}, x) \chi_{r^{\prime}}(\tilde{\omega}, \omega) v_{0, \lambda}(t, \omega, x) d x d \omega d t \\
& =\int_{0}^{T} \int_{\Omega} \eta(x) K_{h}^{*}\left[\chi_{r^{\prime}}(\tilde{\omega}, \cdot) v_{0, \lambda}\right](t, \tilde{\omega}, x) u_{0, \lambda}(t, \tilde{\omega}, x) d x d t .
\end{aligned}
$$

We repeat the same procedure with $r^{\prime} \rightarrow 1$ to obtain $J_{\lambda, r^{\prime}}(\tilde{\omega}) \rightarrow J_{\lambda}(\tilde{\omega})$ a.e. $\tilde{\omega} \in S$, where

$$
\begin{aligned}
J_{\lambda}(\tilde{\omega}) & =\int_{0}^{T} \int_{\Omega} \eta(x) h(\tilde{\omega}, \tilde{\omega}) v_{0, \lambda}(t, \tilde{\omega}, x) u_{0, \lambda}(t, \tilde{\omega}, x) d x d t \\
& =h(\tilde{\omega}, \tilde{\omega}) \int_{0}^{T} \int_{\Omega} \eta(x) \Phi(x-t \tilde{\omega}) \Psi(x-t \tilde{\omega}) d x d t .
\end{aligned}
$$

Now we rewrite the right hand side of (25) as

$$
\begin{aligned}
I_{\lambda, r^{\prime}, r}(\tilde{\omega})=\int_{0}^{T} \int_{Q} \eta\left(K_{h}\left[R_{1, \lambda, r}\right] \chi_{r^{\prime}}(\tilde{\omega}, \cdot) v_{0, \lambda}\right. & \\
& \left.\quad+K_{h}\left[\chi_{r}(\tilde{\omega}, \cdot) u_{0, \lambda}\right] R_{2, \lambda, r^{\prime}}^{*}+K_{h}\left[R_{1, \lambda, r}\right] R_{2, \lambda, r^{\prime}}^{*}\right) d x d \omega d t
\end{aligned}
$$


where $R_{1, \lambda, r}$ is the solution of the initial-boundary value problem

$$
\begin{cases}\partial_{t} R+\omega \cdot \nabla R+q_{1} R & =g_{1} K_{h}[R]+\exp (\mathrm{i} \lambda t) g_{1} z_{1, \lambda, r} \\ R(0, \omega, x) & =0, \quad(\omega, x) \in S \times \Omega \\ R(t, \omega, \sigma) & =0, \quad(\omega, \sigma) \in \Sigma^{-}\end{cases}
$$

with

$$
z_{1, \lambda, r}(t, \tilde{\omega}, \omega, x)=\int_{S} h\left(\omega, \omega^{\prime}\right) \chi_{r}\left(\tilde{\omega}, \omega^{\prime}\right) u_{0, \lambda}\left(t, \omega^{\prime}, x\right) d \omega^{\prime}
$$

and $R_{2, \lambda, r^{\prime}}^{*}$ is the solution of the corresponding adjoint problem with $z_{2, \lambda, r^{\prime}}$.

Again, the properties of $\chi_{r}$ and Lebesgue Theorem give, as $r \rightarrow 1$,

$$
z_{1, \lambda, r} \longrightarrow z_{1, \lambda} \quad \text { in } \quad L^{2}([0, T] \times S \times Q),
$$

where $z_{1, \lambda}(t, \tilde{\omega}, \omega, x)=h(\omega, \tilde{\omega}) u_{0, \lambda}(t, \tilde{\omega}, x)$. From the continuity of the operators $U(t)$ and $K_{h}$ we obtain, as $r \rightarrow 1$,

$$
K_{h}\left[R_{1, \lambda, r}\right](\tilde{\omega}) \longrightarrow K_{h}\left[R_{1, \lambda}\right](\tilde{\omega}) \quad \text { in } \quad C\left([0, T] ; L^{2}(Q)\right)
$$

for almost every $\tilde{\omega} \in S$, where $R_{1, \lambda}$ is the solution of (27) with $z_{1, \lambda}$ taking the place of $z_{1, \lambda, r}$. Therefore, taking the limit as $r \rightarrow 1$, we have $I_{\lambda, r^{\prime}, r}(\tilde{\omega}) \rightarrow I_{\lambda, r^{\prime}}(\tilde{\omega})$ a.e. $\tilde{\omega} \in S$, where

$$
\begin{aligned}
I_{\lambda, r^{\prime}}(\tilde{\omega})= & \int_{0}^{T} \int_{Q} \eta\left(K_{h}\left[R_{1, \lambda}\right] \chi_{r^{\prime}}(\tilde{\omega}, \cdot) v_{0, \lambda}+h(\cdot, \tilde{\omega}) u_{0, \lambda} R_{2, \lambda, r^{\prime}}^{*}\right. \\
& \left.+K_{h}\left[R_{1, \lambda}\right] R_{2, \lambda, r^{\prime}}^{*}\right) d x d \omega d t \\
= & \int_{0}^{T} \int_{Q} \eta\left(K_{h}^{*}\left[\chi_{r^{\prime}}(\tilde{\omega}, \cdot) v_{0, \lambda}\right] R_{1, \lambda}+K_{h}^{*}\left[R_{2, \lambda, r^{\prime}}^{*}\right] R_{1, \lambda}\right) d x d \omega^{\prime} d t+ \\
& +\int_{0}^{T} \int_{\Omega} \eta K_{h}^{*}\left[R_{2, \lambda, r^{\prime}}^{*}\right](t, \tilde{\omega}, x) u_{0, \lambda}(t, \tilde{\omega}, x) d x d t
\end{aligned}
$$

We repeat the same procedure with $r^{\prime} \rightarrow 1$ to obtain $I_{\lambda, r^{\prime}}(\tilde{\omega}) \rightarrow I_{\lambda}^{*}(\tilde{\omega})$ a.e. $\tilde{\omega}$, where

$$
\begin{aligned}
I_{\lambda}^{*}(\tilde{\omega})=\int_{0}^{T} \int_{\Omega} \eta\left(K_{h}\left[R_{1, \lambda}\right] v_{0, \lambda}+K_{h}^{*}\left[R_{2, \lambda}^{*}\right]\right. & \left.u_{0, \lambda}\right) d x d t \\
& +\int_{0}^{T} \int_{Q} \eta K_{h}\left[R_{1, \lambda}\right] R_{2, \lambda}^{*} d x d \omega d t .
\end{aligned}
$$

In order to take the limit as $\lambda \rightarrow+\infty$ in (28), we point out that $x \mapsto e^{\mathrm{i} \lambda \tilde{\omega} \cdot x}$ converges weakly to zero in $L^{2}(\Omega)$ for all $\tilde{\omega} \in S$. Therefore, using the Lebesgue Theorem and the continuity of the operators $U(t)$ and $K_{h}$ we may conclude that

$$
e^{\mathrm{i} \lambda t} g_{1} z_{1, \lambda}(t, \tilde{\omega}, \cdot, \cdot) \rightarrow 0 \Rightarrow R_{1, \lambda}(t, \tilde{\omega}, \cdot, \cdot) \rightarrow 0 \Rightarrow K_{h}\left[R_{1, \lambda}\right](t, \tilde{\omega}, \cdot, \cdot) \rightarrow 0,
$$


weakly in $L^{2}(Q), \forall t \in[0, T]$, a.e. $\tilde{\omega} \in S$.

On another hand, it follows from (12) that $\omega \mapsto R_{1, \lambda}(t, \tilde{\omega}, \omega, x)$ is bounded in $L^{2}(S)$ a.e. in $[0, T] \times Q$. Since $K_{h}$ is compact, we have (by taking a sequence $\lambda_{n}$ if necessary) that $K_{h}\left[R_{1, \lambda}\right](t, \tilde{\omega}, \cdot, x)$ converges strongly in $L^{2}(S)$ as $\lambda \rightarrow+\infty$. As a consequence of the Lebesgue Theorem, $K_{h}\left[R_{1, \lambda}\right](t, \tilde{\omega}, \cdot, \cdot)$ converges strongly in $L^{2}(Q)$, $\forall t \in[0, T]$, a.e. $\tilde{\omega} \in S$. In particular, we have from (29) that

$$
K_{h}\left[R_{1, \lambda}\right](t, \tilde{\omega}, \cdot, \cdot) \longrightarrow 0 \text { strongly in } L^{2}(Q), \forall t \in[0, T] \text {, a.e. } \tilde{\omega} \in S .
$$

With the same arguments, we also obtain

$$
K_{h}\left[R_{2, \lambda}^{*}\right](t, \tilde{\omega}, \cdot, \cdot) \longrightarrow 0 \text { strongly in } L^{2}(Q), \forall t \in[0, T] \text {, a.e. } \tilde{\omega} \in S \text {. }
$$

Since $u_{0, \lambda}$ and $v_{0, \lambda}$ are bounded functions, we see from (30), (31), and (28) that

$$
\lim _{\lambda \rightarrow+\infty} I_{\lambda}^{*}(\tilde{\omega})=0, \quad \text { a.e. } \tilde{\omega} \in S .
$$

Since we have by $(25), J_{\lambda, r^{\prime}, r}(\tilde{\omega})=I_{\lambda, r^{\prime}, r}(\tilde{\omega})$ a.e. $\tilde{\omega} \in S$, we take the limit in $r^{\prime}, r$ and $\lambda$ and we get, from (26), (32), and the assumption that $h(\tilde{\omega}, \tilde{\omega}) \neq 0$, that

$$
\int_{0}^{T} \int_{\mathbb{R}^{N}} \eta(x+t \tilde{\omega}) \Phi(x) \Psi(x) d x d t=\int_{0}^{T} \int_{\Omega} \eta(x) \Phi(x-t \tilde{\omega}) \Psi(x-t \tilde{\omega}) d x d t=0 .
$$

Since $\Phi$ and $\Psi$ are arbitrary and $T>\operatorname{diam}(\Omega)$, we obtain

$$
\int_{-\infty}^{+\infty} \eta(x+t \tilde{\omega}) d t=0, \quad \text { a.e. } x \in \mathbb{R}^{N}, \tilde{\omega} \in S
$$

which means, by standard arguments for the X-ray transform, that $\eta(x)=0$ a.e. in $\mathbb{R}^{N}$. Hence the result.

\section{References}

[1] A.-P. Calderón, On an inverse boundary value problem, Seminar on Numerical Analysis and its Applications to Continuum Physics (Rio de Janeiro, 1980), 1980, pp. 65-73.

[2] T. Cazenave and A. Haraux, Equations d'évolution linéaires: Semi-groupes, applications e approximations, DEA d'Analyse Numérique 1995-1996.

[3] M. Cessenat, Théorèmes de trace $L^{p}$ pour des espaces de fonctions de la neutronique, C. R. Acad. Sci. Paris Sér. I Math. 299 (1984), no. 16, 831-834 (French, with English summary).

[4] Théorèmes de trace pour des espaces de fonctions de la neutronique, C. R. Acad. Sci. Paris Sér. I Math. 300 (1985), no. 3, 89-92 (French, with English summary).

[5] M. Choulli and P. Stefanov, Inverse scattering and inverse boundary value problems for the linear Boltzmann equation, Comm. Partial Differential Equations 21 (1996), no. 5-6, 763-785.

[6] R. Cipolatti and I. F. Lopez, Determination of coefficients for a dissipative wave equation via boundary measurements, J. Math. Anal. Appl. 306 (2005), no. 1, 317-329. 
[7] R. Dautray and J.-L. Lions, Mathematical analysis and numerical methods for science and technology. Vol. 6, Springer-Verlag, Berlin, 1993.

[8] G. B. Folland, Introduction to partial differential equations, Mathematical Notes, Princeton University Press, Princeton, N.J., 1976.

[9] V. Isakov, Inverse problems for partial differential equations, Applied Mathematical Sciences, vol. 127, Springer-Verlag, New York, 1998.

[10] A. Louis and F. Natterer, Mathematical problems of computarized tomography, Procc. IEEE 71 (1983), no. 1, 379-389.

[11] M. Mokhtar-Kharroubi, Mathematical topics in neutron transport theory, Series on Advances in Mathematics for Applied Sciences, vol. 46, World Scientific Publishing Co. Inc., River Edge, NJ, 1997.

[12] Rakesh and W. W. Symes, Uniqueness for an inverse problem for the wave equation, Comm. Partial Differential Equations 13 (1988), no. 1, 87-96.

[13] M. Reed and B. Simon, Methods of modern mathematical physics. III, Academic Press, New York, 1979.

[14] V. G. Romanov, Estimation of stability in the problem of determining the attenuation coefficient and the scattering indicatrix for the transport equation, Sibirsk. Mat. Zh. 37 (1996), no. 2, 361-377 (Russian); English transl., Siberian Math. J. 37 (1996), no. 2, 308-324.

[15] _ Stability estimates in the three-dimensional inverse problem for the transport equation, J. Inverse Ill-Posed Probl. 5 (1997), no. 5, 463-475.

[16] P. Stefanov and G. Uhlmann, Optical tomography in two dimensions, Methods Appl. Anal. 10 (2003), no. 1, 1-9.

[17] A. Tamasan, An inverse boundary value problem in two-dimensional transport, Inverse Problems 18 (2002), no. 1, 209-219.

[18] J.-N. Wang, Stability estimates of an inverse problem for the stationary transport equation, Ann. Inst. H. Poincaré Phys. Théor. 70 (1999), no. 5, 473-495. 